\title{
Mid-Infrared Generation by Frequency Down-Conversion Across 1.2 Octaves in a Normally-Dispersive Silicon Wire
}

\author{
Bart Kuyken ${ }^{1}$, Peter Verheyen ${ }^{2}$, Pamela Tannouri ${ }^{3}$, Joris Van Campenhout ${ }^{2}$, Roel Baets ${ }^{1}$, Gunther \\ Roelkens $^{1}$, and William M. J. Green ${ }^{3}$ \\ ${ }^{I}$ Photonics Research Group, Department of Information Technology, Ghent University - imec, Ghent B-9000, Belgium \\ ${ }^{2}$ imec, Kapeldreef 75, Leuven B-3001, Belgium \\ ${ }^{3}$ IBM T. J. Watson Research Center, 1101 Kitchawan Road, Yorktown Heights, NY 10598, USA. \\ Tel.:+32 (0)9 32-9-264-3335. Email: Bart.Kuyken@intec.ugent.be
}

\begin{abstract}
Using four-wave mixing in normally-dispersive mid-infrared silicon nanophotonic waveguides, we demonstrate the conversion of light across more than 1.2 octaves, from the telecom band to a wavelength of $3630 \mathrm{~nm}$.

(C) 2013 Optical Society of America

OCIS codes: (190.4380) Nonlinear optics, four-wave mixing; (190.4390) Nonlinear optics, integrated optics
\end{abstract}

\section{Introduction}

The long-wavelength transparency of the silicon-on-insulator (SOI) wire waveguide platform up to $\sim 4 \mu \mathrm{m}$ [1], limited by the absorption of the silicon oxide, has recently been leveraged to expand the application of the platform beyond the traditional telecom space. A new set of mid-infrared spectroscopic applications arise from the specific and strong absorption lines of many molecules within this "molecular fingerprinting" wavelength region [2], which enable the development of chip-scale optical sensors with high sensitivity and high selectivity. Previous work has exploited the intrinsically high third-order nonlinearity and strong optical confinement of silicon wire waveguides to generate the mid-infrared radiation required for such chip-scale sensors. Recent demonstrations have shown that four-wave mixing-based nonlinear optical functions including supercontinuum generation [3], optical parametric amplification [4,5], and wavelength conversion [6-8], can be integrated in compact mid-infrared silicon photonic integrated circuits. In this paper, we demonstrate generation of midinfrared radiation near $3630 \mathrm{~nm}$ by mixing a high-power pump at $2190 \mathrm{~nm}$ with a telecom signal at $1564 \mathrm{~nm}$, within a silicon photonic wire engineered to have normal dispersion. Simultaneously, we illustrate that the telecom band signal is amplified by up to $13.1 \mathrm{~dB}$ for a peak pump power of $18.3 \mathrm{~W}$. To the best of our knowledge, this is the first time that wavelength conversion across more than an octave has been demonstrated in silicon waveguides.

\section{Phase-matching in a silicon waveguide}

The phase-matching condition for the degenerate four-wave mixing process in a waveguide is given by

$$
2^{2}+\frac{1}{12} \quad 4^{4}+2 \quad P=0
$$

Here, $\gamma$ is the effective nonlinearity parameter of the waveguide, $\Delta \omega$ is the frequency detuning between pump and signal (or idler), $\beta_{2}$ and $\beta_{4}$ are the second- and fourth-order dispersion coefficients respectively, evaluated at the pump frequency, and $P$ is the pump peak power. The higher-order dispersion terms are necessary when conversion over a wide band is envisaged. In this particular photonic wire the second-order dispersion is normal, e.g. positive, such that the fourth-order dispersion $\beta_{4}$ has to be negative to achieve phase-matching. This facilitates phase-matching far from the pump [9]. For an air-clad wire waveguide $1650 \mathrm{~nm}$ wide and $400 \mathrm{~nm}$ thick (as shown in the inset of Fig. 1, having $\gamma=20 \mathrm{~W}^{-1} \mathrm{~m}^{-1}$ ), the phase-matched idler and signal solutions of Eq. (1) are plotted in Fig. 1, for pump wavelengths between $2100 \mathrm{~nm}$ and $2400 \mathrm{~nm}$. A fixed pump peak power of $20 \mathrm{~W}$ is assumed. Pumping within the normal dispersion regime at $2190 \mathrm{~nm}$, the simulation in Fig. 1 predicts that an input signal near $1700 \mathrm{~nm}$ can be down-converted to a band near $3076 \mathrm{~nm}$.

\section{Experimental results}

The $1 \mathrm{~cm}$-long silicon nanophotonic wire used in the experiment is fabricated in imec's CMOS pilot line, on a $200 \mathrm{~mm}$ SOI wafer consisting of a $400 \mathrm{~nm}$ silicon waveguide layer on a $2 \mu \mathrm{m}$ buried oxide. The dimensions of the photonic wire match those shown in the inset of Fig. 1. For the experiment, a picosecond pulse train (FWHM $\sim 2 \mathrm{ps}$, repetition rate $=76 \mathrm{MHz}$ ) generated by a tunable optical parametric oscillator (OPO) is coupled to a single-mode optical fiber, and used as the pump. A telecom tunable $\mathrm{CW}$ laser is used as a probe. Coupling into/out of the SOI photonic wire is accomplished using lensed fibers. Signal and pump are combined by a 90/10 fused silica fiber coupler. A polarization controller is used to excite the TE polarized waveguide modes of both the pump and the probe. The waveguide loss is measured at $<0.2 \mathrm{~dB} / \mathrm{cm}$ at both the telecom and pump wavelengths. The fiber coupling loss at each facet is $8.5 \mathrm{~dB} \pm 1 \mathrm{~dB}$. The waveguide output spectrum up to 2500 
$\mathrm{nm}$ is characterized with a mid-infrared optical spectrum analyzer, at $1 \mathrm{~nm}$ spectral resolution. For spectral analysis at wavelengths longer than $2500 \mathrm{~nm}$, a Fourier Transform Infrared spectrometer (FTIR) equipped with a liquid-nitrogen cooled InAs detector is used. In this case, the light is coupled out of the waveguide and collimated into the FTIR with a high numerical aperture (NA) chalcogenide lens.

In a first experiment, the peak wavelength of the spontaneous parametric fluorescence (modulation instability: MI) excited by the high peak power pulses was recorded as a function of the input pump wavelength. The MI peaks indicate the wavelength bands at which phase-matching is achieved. The wavelengths at which the MI peaks appear on the blue side of the pump are shown as the red triangles in Fig. 1, as a function of pump wavelength. The red stars correspond to the energy-conserving wavelengths on the red side of the pump. As illustrated by the dashed line in Fig. 1, for a pump tuned to $2190 \mathrm{~nm}$ we expect phase-matching between signals near $1700 \mathrm{~nm}$ and $3076 \mathrm{~nm}$. Experimentally, an input probe signal at $1565 \mathrm{~nm}$ is phase-matched for efficient wavelength conversion to $3635 \mathrm{~nm}$. Fig. 2 (a) shows the optical spectrum at the waveguide output in this case, as recorded by the FTIR. A long pass filter is used at the FTIR input to suppress the transmitted pump pulses. In addition to the residual $2190 \mathrm{~nm}$ pump, Fig. 2 (a) also exhibits a converted idler peak at $3630 \mathrm{~nm}$ protruding above the thermal background radiation floor. Figure 2 (b) plots the on-chip parametric gain as a function of the telecom band signal wavelength, for a pump wavelength of $2190 \mathrm{~nm}$ and a peak pump power of $18.3 \mathrm{~W}$. Following the measurement procedure outlined in [4], the figure shows that net gain is observed from $1550 \mathrm{~nm}$ up to $1585 \mathrm{~nm}$, with a peak value of $13.1 \mathrm{~dB}$ at $1564.4 \mathrm{~nm}$. The conversion gain at $3635 \mathrm{~nm}$ is estimated to be $9.2 \mathrm{~dB}$ according to the Manley-Rowe relations. The lack of an appropriate tunable laser source prevents a similar set of measurements for up-conversion of wavelengths near $3635 \mathrm{~nm}$ to the telecom band.

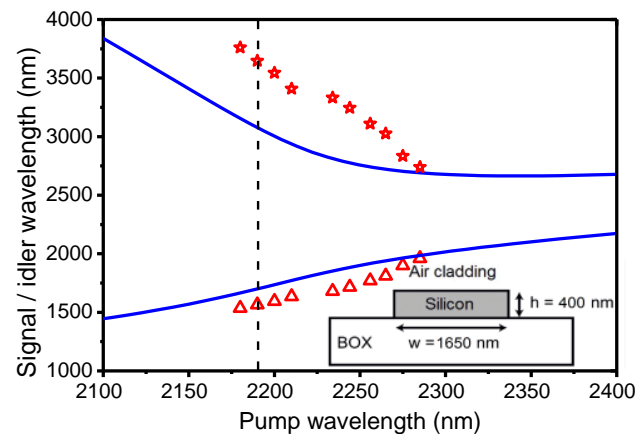

Figure 1: Phase-matched idler and signal wavelength as a function of pump wavelength. The blue curve shows the simulated phase-matched wavelengths, for a peak pump power of $20 \mathrm{~W}$. The red triangles label the positions of the experimentally observed modulation instability peaks on the blue side of the pump, while the red stars correspond to the energy-conserving wavelengths on the red side of the pump.
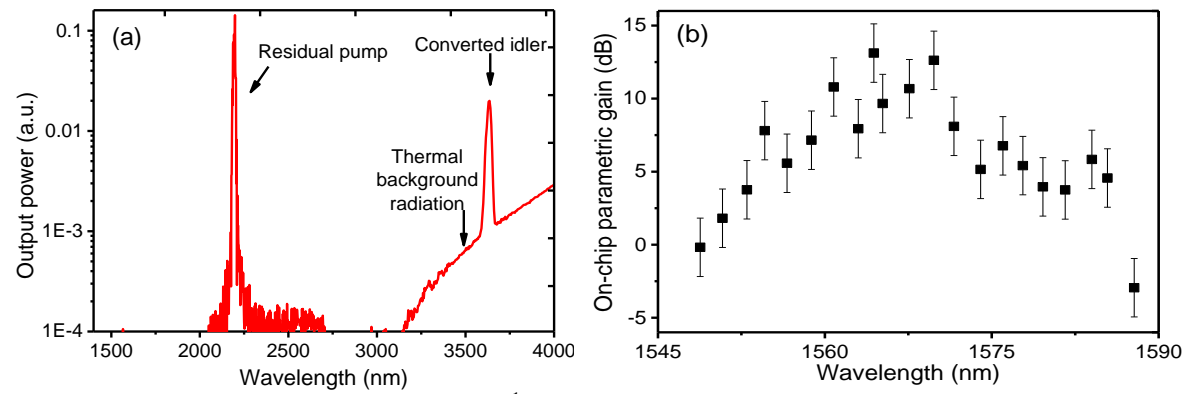

Figure 2: (a) Optical spectrum recorded with an FTIR $\left(16 \mathrm{~cm}^{-1}\right.$ resolution) when the waveguide is pumped at $2190 \mathrm{~nm}$ with a peak power of $18.3 \mathrm{~W}$, and probed by a telecom signal at $1565 \mathrm{~nm}$. The converted idler is seen at $3635 \mathrm{~nm}$, above the thermal background radiation signal

(b) On-chip gain for the telecom CW probe as a function of wavelength in the $1 \mathrm{~cm}$-long silicon wire waveguide for $18.3 \mathrm{~W}$ pump pulses

centered at $2190 \mathrm{~nm}$. Error bars originate from the $+/-1 \mathrm{~dB}$ uncertainty in the value of the pump power coupled into the waveguide.

\section{Acknowledgements}

This work was supported by the FP7-ERC-MIRACLE and FP7-ERC-InSpectra project. Bart Kuyken acknowledges a scholarship provided by the Fund for Scientific Research Flanders (FWO-Vlaanderen).

[1] M. Milosevic, et al., "Silicon waveguides and devices for the mid-infrared," Applied Physics Letters 101, 121105-121105 (2012).

[2] J. G. Crowder, et al., "Infrared methods for gas detection," in Mid-Infrared Semiconductor Optoelectronics. New York: Springer-Verlag, 2006.

[3] B. Kuyken, et al., "Mid-infrared to telecom-band supercontinuum generation in highly nonlinear silicon-on-insulator wire waveguides," Optics Express 19, 20172-20181 (2011).

[4] X. Liu, et al., "Mid-infrared optical parametric amplifier using silicon nanophotonic waveguides," Nature Photonics 4, 557-560 (2010)

[5] B. Kuyken, et al., "50 dB parametric on-chip gain in silicon photonic wires," Optics Letters 36, 4401-4403 (2011).

[6] S. Zlatanovic, et al., "Mid-infrared wavelength conversion in silicon waveguides using ultracompact telecom-band-derived pump source," Nature Photonics 4, 561-564 (2010).

[7] Q. Lin, et al., "A proposal for highly tunable optical parametric oscillation in silicon micro-resonators," Optics Express 16, 10596-10610 (2008).

[8] X. Liu, et al., "Bridging the mid-infrared-to-telecom gap with silicon nanophotonic spectral translation," Nature Photonics 6, 667-669 (2012).

[9] R.K. Lau, et al., "Continuous-wave mid-infrared frequency conversion in silicon nanowaveguides," Optics Letters 36, 1263-1265 (2011). 